\title{
Multisensor Multi-target Tracking
}

\author{
Mike Ignacio, James Duang
}

\begin{abstract}
In a normal multitarget tracking (MTT) situation, the sensor state is either accepted known, or following is acted in the sensor's (relative) organize outline. This supposition doesn't hold when the sensor, e.g., a car radar, is mounted on a vehicle, and the objective state ought to be spoken to in a worldwide (outright) organize outline. At that point it is essential to consider the questionable area of the vehicle on which the sensor is mounted for MTT.

In this paper, we present a multisensor low unpredictability Poisson multi-Bernoulli MTT channel, which together tracks the questionable vehicle state and target states. Estimations gathered by various sensors mounted on different vehicles with shifting area vulnerability are fused consecutively dependent on the appearance of new sensor estimations. In doing as such, targets saw from a sensor mounted on an all around limited vehicle diminish the state vulnerability of other inadequately confined vehicles, gave that a typical non-void subset of targets is watched. A low multifaceted nature channel is acquired by approximations of the joint sensor-include state thickness limiting the KullbackLeibler divergence (KLD).

Results from engineered just as test estimation information, gathered in a vehicle driving situation, exhibit the presentation advantages of joint vehicle-target state following.
\end{abstract}

\section{INTRODUCTION}

Intelligent transportation systems (ITSs) in general, and autonomous driving $(\mathrm{AD})$ in particular, require accurate position information [1]. Measurements provided by various onboard sensors allow to infer the vehicle state, e.g., position and velocity, as well as information about the surrounding environment. For instance, a global navigation satellite system (GNSS) receiver provides absolute position, whereas a radar sensor provides relative position of a feature with respect to (w.r.t.) the sensor origin. Furthermore, vehicles have access to a pre-recorded local dynamic map (LDM) containing static features such as, e.g., landmarks [2]. Dynamic features such as pedestrians, cyclists, etc., are not part of the pre-recorded map. For an AD system to be fully aware of the surrounding environment, dynamic features need to be estimated from measurements and tracked over time, using the vehicle's onboard sensors, thus allowing to enrich the vehicle's LDM.

In order to update the LDM by new features (static, dynamic) described in a global coordinate frame, location uncertainty of the platform, where the sensors are mounted (e.g., a vehicle), needs to be considered. Furthermore, information from one vehicle can be utilized to increase location accuracy of other vehicles, and vice versa [3], [4]. In the literature, three different research tracks can be discerned: (i) MTT when both features and sensors are mobile, but the sensor states are known; (ii) simultaneous localization and mapping (SLAM) when the sensor state and feature state are unknown, but features are static; (iii) simultaneous localization and tracking (SLAT) combines MTT and SLAM by considering unknown mobile feature and mobile sensor states. All three tracks include measurements due to clutter; missed detections; unknown measurement-to-target correspondence; and target appearance and disappearance.

MTT filters based on random finite set (RFS) and finite-set statistics (FISST), see, e.g., [5], [6] for recent works and [7], [8] for earlier works, have gained much attention. For example, the probability hypothesis density (PHD) filter propagates the first moment of the RFS density over time [9]-[11]. The Poisson multi-Bernoulli mixture (PMBM) filter models unknown, i.e., never detected, features by a Poisson process and detected features by a multi-Bernoulli mixture (MBM) [12]. Based on this, the track-oriented marginal multiple target multiBernoulli/Poisson (TOMB/P) filter approximates the global joint data association (DA) by the product of marginal DAs, similar to the joint probability data association (JPDA) filter, which allows a computationally efficient implementation [12]. In [13], a derivation of the PMBM filter based on standard single target measurement models, without using probability generating functionals (p.g.fl.s) and functional derivatives, is presented. Furthermore, connections to the $\delta$-generalized labeled multi-Bernoulli ( $\delta$-GLMB) filter [14], [15] are discussed. In contrast to the above FISST approaches, [16], [17] proposed a factor graph (FG) based approach for a variant of the JPDA filter. A multi-scan scenario was considered, and the filter was realized by running loopy belief propagation on the FG containing cycles. Additionally, similarities between the FG approach and the TOMB/P filter are discussed.

For static features (called landmarks) observed by a sensor with unknown state, SLAM based methods can be employed to jointly estimate the sensor state and the landmarks (see, e.g., [2], [18]). In [19] and [20], an RFS based approach to the SLAM problem was proposed. The landmark state is conditioned on the sensor location and then tracked through a PHD filter following a Rao-Blackwellization (RB).

Joint estimation of the unknown sensor and mobile feature states, also termed SLAT, was for instance addressed in [21], where a combination of static and mobile features are estimated through MTT. In doing so, a particle based representation of the sensor state is combined with a parametric formulation of the PHD filter. Related to this, [22], [23] considers the problem of sensor state estimation through target tracking in the RFS framework by combining local PHD filters with the help of belief propagation. To achieve distributed processing, approximations in terms of separable likelihoods are taken. In [24], a particle based MTT filter is presented for SLAT in wireless sensor networks. Here, the measurementto-target DA is known, but corrupted by noise leading to false alarms. In [25], a message passing based distributed multisensor MTT filter modeling target and sensor states by Gaussian probability density functions (PDFs) is presented. Measurement-to-target DA is known and targets are always 
present and no false alarm measurements occur. Similar to [17], a FG based approach was considered in [33] for an urban ITS scenario, where the number of features is assumed a priori known, and in [4] where the DA assumed to be known as well.

In this paper, we consider the problem of multisensor SLAT for joint estimation of the unknown sensor and feature states, enabling accurate feature tracking w.r.t. the sensor uncertainty. Our proposed MTT filter builds upon the Bayesian RFS based PMBM filter [12] with proper feature birth and death processes, but explicitly models the sensor state uncertainty. A low complexity filter is obtained by approximations of the joint sensor-feature state density minimizing the KLD. A tractable implementation is achieved through approximations similar to the TOMB/P filter [12]. The proposed MTT filter allows to track the state of the sensor platform not only by (direct) measurements of the platform itself, but also through feature tracking in a multi-sensor setup. The main contributions are:

- A low-complexity asynchronous multisensor MTT filter with uncertain sensor state information,

- Sensor state tracking by fusion of multisensor MTT information with local sensor information,

- Validation of the filter with real sensor data, as well as in-depth analysis of performance with synthetic data.

The remainder of this paper is organized as follows; Section II gives some background knowledge on RFS, and Section III introduces the problem formulation and system models. Section IV details the proposed MTT filter with uncertain sensor state, including the multisensor generalization of the proposed MTT filter. Results with synthetic data are given in Section $\mathrm{V}$ and with experimental data in Section VI, respectively. Conclusions are drawn in Section VII.

\section{Notation}

Scalars are described by non-bold letters $r$, vectors by lower-case bold letters $\boldsymbol{x}$; matrices and sets by upper-case bold letters $\boldsymbol{X}$. The cardinality of set $\boldsymbol{X}$ is denoted $|\boldsymbol{X}|$. The set operator $\uplus$ denotes the disjoint set union, i.e., $\boldsymbol{X}^{u} \uplus \boldsymbol{X}^{d}=\boldsymbol{X}$ means $\boldsymbol{X}^{u} \cup \boldsymbol{X}^{d}=\boldsymbol{X}$ and $\boldsymbol{X}^{u} \cap \boldsymbol{X}^{d}=\emptyset$. The vehicle/sensor state is reserved by letter $s$, the feature state by letter $\boldsymbol{x}$, and measurements by letter $\boldsymbol{z}$. The identity matrix of size $n \times n$ is denoted $\boldsymbol{I}_{n}$. The $\ell_{2}$-norm of vector $\boldsymbol{x}$ is $\|\boldsymbol{x}\|_{2}$.

\section{BACKGROUND ON RFS}

In this section, we describe some useful properties of an RFS. If not stated otherwise, the source of all these is [12].

\section{A. Random Finite Set Formulation}

According to [12], RFS based methods have been developed in [5] to conduct statistical inference in problems in which the variables of interest and/or observations form finite sets. In tracking, they address two major challenges of interest: (i) the number of targets present in the scene is unknown, (ii) measurements are invariant to ordering (measurement-totarget correspondence is unknown). An RFS $\boldsymbol{X}$ is a finite-set valued random variable, which can be described by a discrete probability distribution $p(n)$, where $n \geq 0$ denotes the number of elements $\boldsymbol{x}_{i} \in \boldsymbol{X}$ for $i=0, \ldots, n$ and a family of joint PDFs $p_{n}\left(\boldsymbol{x}_{1}, \ldots, \boldsymbol{x}_{n}\right)$ yielding [12]

$$
f\left(\left\{\boldsymbol{x}_{1}, \ldots, \boldsymbol{x}_{n}\right\}\right)=p(n) \sum_{\pi} p_{n}\left(\boldsymbol{x}_{\pi(1)}, \ldots, \boldsymbol{x}_{\pi(n)}\right),
$$

where the sum spans over the $n$ ! permutation functions $\pi(\cdot)$, such that its RFS density $f(\boldsymbol{X})$ is permutation invariant. The set integral of a real-valued function $g(\boldsymbol{X})$ of a finite-set variable $\boldsymbol{X}$ is defined as [5, p. 361], [12]

$$
\int g(\boldsymbol{X}) \delta \boldsymbol{X} \triangleq g(\emptyset)+\sum_{n=1}^{\infty} \frac{1}{n !} \int g\left(\left\{\boldsymbol{x}_{1}, \ldots, \boldsymbol{x}_{n}\right\}\right) \mathrm{d} \boldsymbol{x}_{1} \cdots \mathrm{d} \boldsymbol{x}_{n}
$$

Two important examples of RFSs are Bernoulli processes (and their generalization, multi-Bernoulli (MB) processes) and Poisson processes. A Bernoulli process $\boldsymbol{X}$ with probability of existence $r$ and existence-conditioned PDF $p(\boldsymbol{x})$ has RFS density

$$
f(\boldsymbol{X})= \begin{cases}1-r, & \text { if } \boldsymbol{X}=\emptyset \\ r \cdot p(\boldsymbol{x}), & \text { if } \boldsymbol{X}=\boldsymbol{x} \\ 0, & \text { otherwise }\end{cases}
$$

The RFS density of a multi-Bernoulli (MB) process $\boldsymbol{X}$ can be expressed as

$$
f(\boldsymbol{X})=\sum_{\uplus_{i \in \mathbb{I}} \boldsymbol{X}_{i}=\boldsymbol{X}} \prod_{i \in \mathbb{I}} f_{i}\left(\boldsymbol{X}_{i}\right)
$$

for $|\boldsymbol{X}| \leq|\mathbb{I}|$, and $f(\boldsymbol{X})=0$ otherwise. Here, $\mathbb{I}$ is the index set of the MB with components $\left\{r_{i}, p_{i}(\boldsymbol{x})\right\}_{i \in \mathbb{I}}$. A MBM is a linear combination of MBs with density expressed by the Bernoulli components

$$
f(\boldsymbol{X})=\sum_{j \in \mathbb{J}} w_{j} f_{j}(\boldsymbol{X}),
$$

where $w_{j}$ is the weight of the $j$-th MB with density $f_{j}(\boldsymbol{X})$ (such that $\sum_{j} w_{j}=1$ ), and $\mathbb{J}$ is the index set of the MBM. A Poisson point process (PPP) with intensity function $\lambda_{c}(\boldsymbol{y})$ has RFS density [12]

$$
f(\boldsymbol{Y})=e^{-\left\langle\lambda_{c}, 1\right\rangle} \prod_{\boldsymbol{y} \in \boldsymbol{Y}} \lambda_{c}(\boldsymbol{y})
$$

with inner product $\left\langle\lambda_{c}, h\right\rangle \triangleq \int \lambda_{c}(\boldsymbol{y}) h(\boldsymbol{y}) \mathrm{d} \boldsymbol{y}$.

Remark 1: If $\boldsymbol{X}$ and $\boldsymbol{Y}$ are independent RFSs such that $\boldsymbol{Z}=\boldsymbol{X} \uplus \boldsymbol{Y}$, then

$$
f_{\boldsymbol{Z}}(\boldsymbol{Z})=\sum_{\boldsymbol{X} \uplus \boldsymbol{Y}=\boldsymbol{Z}} f_{\boldsymbol{X}}(\boldsymbol{X}) f_{\boldsymbol{Y}}(\boldsymbol{Y}) .
$$

Note, when $\boldsymbol{X}$ follows an MBM process and $\boldsymbol{Y}$ an PPP (7) is called a PMBM density and Poisson multi-Bernoulli (PMB) density for $|\mathbb{J}|=1$.

Remark 2: A common way to estimate the set states from a Bernoulli process with RFS density $f(\boldsymbol{X})$ is by comparing the probability of existence $r$ to an existence threshold $r_{\mathrm{th}}$. For $r>r_{\text {th }}$, the target is said to exist and has PDF $p(\boldsymbol{x})$ (c.f. (3)). Its state can then be estimated by the mean of $p(\boldsymbol{x})$, i.e., $\hat{\boldsymbol{x}}=\int \boldsymbol{x} p(\boldsymbol{x}) \mathrm{d} \boldsymbol{x}$. See [13, Sec. VI], for an extended discussion on state estimation. 


\section{B. Bayesian Filter Formulation}

Similar to the random vector (RV) case, an RFS based filter can be described, conceptually at least, within the Bayesian framework with alternating prediction and update steps operating on the state RFS $\boldsymbol{X}$ with [5, Ch. 14]

$$
f_{+}(\boldsymbol{X})=\int f\left(\boldsymbol{X} \mid \boldsymbol{X}^{\prime}\right) f_{-}\left(\boldsymbol{X}^{\prime}\right) \delta \boldsymbol{X}^{\prime}
$$

and

$$
f(\boldsymbol{X} \mid \boldsymbol{Z}) \propto \ell(\boldsymbol{Z} \mid \boldsymbol{X}) f_{+}(\boldsymbol{X}),
$$

where $f_{-}\left(\boldsymbol{X}^{\prime}\right)$ is the prior RFS density, $f\left(\boldsymbol{X} \mid \boldsymbol{X}^{\prime}\right)$ is the RFS transition density, $f_{+}(\boldsymbol{X})$ is the predicted RFS density, and $\ell(\boldsymbol{Z} \mid \boldsymbol{X})$ is the RFS measurement likelihood for measurement set $Z$.

\section{Two Useful Lemmas}

Lemma 1: A joint density $f(\boldsymbol{X}, \boldsymbol{Y})$ is approximated in the minimum KLD sense by [34, Ch. 10]

$$
f(\boldsymbol{X}, \boldsymbol{Y}) \approx \int f(\boldsymbol{X}, \boldsymbol{Y}) \delta \boldsymbol{X} \int f(\boldsymbol{X}, \boldsymbol{Y}) \delta \boldsymbol{Y}
$$

Lemma 2: The set integral can be expressed as [35, Lemma 5]

$$
\int \sum_{\boldsymbol{X} \uplus \boldsymbol{Y}=\boldsymbol{X}} f(\boldsymbol{X}) g(\boldsymbol{Y}) \delta \boldsymbol{X}=\int f(\boldsymbol{X}) \delta \boldsymbol{X} \int g(\boldsymbol{Y}) \delta \boldsymbol{Y} .
$$

\section{Motivation, Problem Formulation And System MODELS}

Here, we first present the motivation and problem formulation, as well as the vehicle and feature dynamics. This is followed by the GNSS and vehicle-to-feature (V2F) measurement models, and the communication assumption.

\section{A. Motivation}

We consider an urban ITS scenario, consisting of cooperating vehicles (illustrated in Fig. 1). Each vehicle is equipped with a GNSS-type receiver to determine its absolute position and radar-type sensor to retrieve relative positions of mobile features present in the environment through V2F measurements. Our goal is to develop a filter, which runs on an road side unit (RSU), to track the features and the states of all vehicles in every discrete time step $t$ through incorporation of all measurements provided by the vehicle's on-board GNSS and V2F sensors up until time step $t$.

\section{B. Vehicle and Feature Dynamics}

Vehicle state motion follows independent identically distributed (IID) Markovian processes, where the time-varying vehicle state $\boldsymbol{s}_{v, t}$ of each vehicle $v \in \mathcal{V}$ at time step $t$ is statistically modeled as $p\left(\boldsymbol{s}_{v, t} \mid \boldsymbol{s}_{v, t-1}\right)$. Each feature $k \in \mathcal{K}$ with state $\boldsymbol{x}_{k, t-1}$ survives to the next time step $t$ following an IID Markovian process with survival probability $p_{S}\left(\boldsymbol{x}_{k, t}\right)$. The feature state motion follows IID Markovian processes and is statistically modeled as $p\left(\boldsymbol{x}_{k, t} \mid \boldsymbol{x}_{k, t-1}\right)$. Note that vehicle and

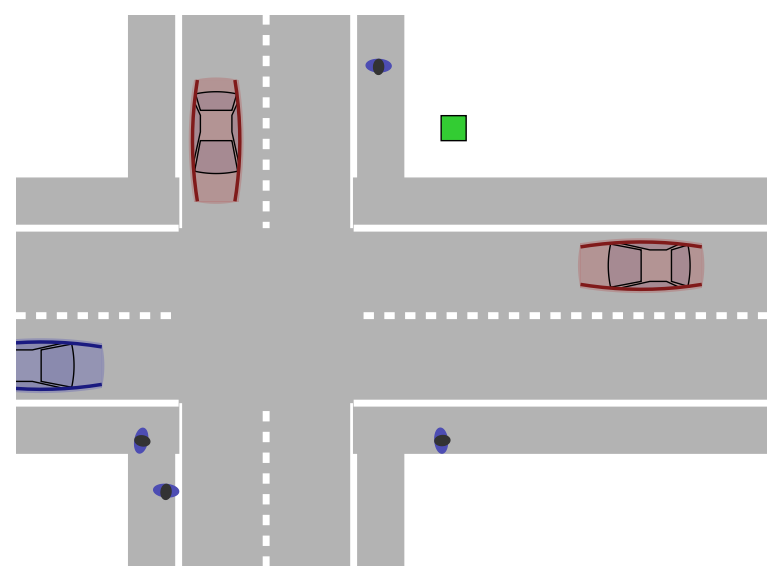

Fig. 1. Urban ITS scenario with two vehicles (red color) cooperating through the RSU (green color) and five mobile features (blue color).

feature state motion are independent. ${ }^{1}$ In the following, we will drop the subscript indexing on states and measurements w.r.t. vehicle/feature/time whenever the context allows.

\section{Measurement Models}

At time step $t$, vehicle $v \in \mathcal{V}$ obtains two different kinds of measurements: (i) measurements of the vehicle state $s$ w.r.t. the reference frame, i.e., GNSS-like measurements $z_{\mathrm{G}}$ modeled by $p\left(\boldsymbol{z}_{\mathrm{G}} \mid \boldsymbol{s}\right)$; and (ii) measurements w.r.t. to features, i.e., from a radar-like (on-board) V2F sensor. Without loss of generality, we assume that the on-board sensors' state is equal to the vehicle state. Furthermore, the measurement-tofeature state correspondence is not known and we assume that the standard MTT measurement assumptions for point targets apply, i.e., each feature generates at most one measurement per measurement scan and sensor, and each measurement has a unique source (feature or clutter).

Let $\boldsymbol{Z}$ be a set of V2F measurements taken by a vehicle at a certain time. This set can be expressed as $\boldsymbol{Z}=\boldsymbol{Z}^{\mathrm{FA}} \uplus \boldsymbol{Z}^{\mathrm{D}}$, where $Z^{\mathrm{FA}}$ denotes the set of false alarm measurements due to clutter (modeled by a PPP with intensity $\lambda_{c}(\boldsymbol{z})$ ) and $\boldsymbol{Z}^{\mathrm{D}}$ denotes the set of feature measurements. Let a V2F measurement $z \in Z^{\mathrm{D}}$ be obtained through the V2F sensor with likelihood function $g(\boldsymbol{z} \mid \boldsymbol{s}, \boldsymbol{x})$. The likelihood for measurement set $\boldsymbol{Z}=\left\{\boldsymbol{z}_{1}, \ldots, \boldsymbol{z}_{m}\right\}$ is defined as in [13, Eqn. 13] by

$$
\begin{aligned}
l(\boldsymbol{Z} \mid \boldsymbol{X}, \boldsymbol{s}) & =e^{-\left\langle\lambda_{c} ; 1\right\rangle} \sum_{\boldsymbol{U} \uplus \boldsymbol{X}_{1} \ldots \uplus \boldsymbol{X}_{m}=\boldsymbol{X}}\left[1-p_{D}(\boldsymbol{s}, \cdot)\right]^{\boldsymbol{U}} \\
& \times \prod_{i=1}^{m} \tilde{l}\left(\boldsymbol{z}_{i} \mid \boldsymbol{X}_{i}, \boldsymbol{s}\right)
\end{aligned}
$$

with

$$
\tilde{l}(\boldsymbol{z} \mid \boldsymbol{X}, \boldsymbol{s})= \begin{cases}p_{D}(\boldsymbol{s}, \boldsymbol{x}) g(\boldsymbol{z} \mid \boldsymbol{s}, \boldsymbol{x}) & \boldsymbol{X}=\{\boldsymbol{x}\} \\ \lambda_{c}(\boldsymbol{z}) & \boldsymbol{X}=\emptyset \\ 0 & |\boldsymbol{X}|>1\end{cases}
$$

\footnotetext{
${ }^{1}$ Note that the proposed filter only predicts over small time-horizons in the order of a few tens of milliseconds. Then the assumption on vehicle and feature states evolving independently is reasonable, because there is very little interaction among them within the prediction horizon.
} 
Here, the feature set has been decomposed into all possible sets $\boldsymbol{U} \uplus \boldsymbol{X}_{1} \ldots \uplus=\boldsymbol{X}$, where the set $\boldsymbol{U}$ represents unknown features and sets $\boldsymbol{X}_{i}$ represent the origin of the $i$ th measurement, which can either be a singleton containing the state of the feature that gave rise to the measurement or an empty set if the measurement is clutter, i.e., $\left|\boldsymbol{X}_{i}\right| \leq 1 \forall i$. Above, $p_{\mathrm{D}}(\boldsymbol{s}, \boldsymbol{x})$ denotes the probability of detection, which depends on the vehicle state $s$ as well as on the feature state $\boldsymbol{x}$. For instance, a limited sensor field-of-view (FoV) affects the probability of feature detection based on the distance between vehicle and feature. An alternative, but equivalent form to represent the likelihood (12), is [13, Eqns. 25, 26]

$$
\begin{aligned}
l_{o}\left(\boldsymbol{Z} \mid \boldsymbol{U}, \boldsymbol{X}_{1}, \ldots, \boldsymbol{X}_{n}, \boldsymbol{s}\right) & =\sum_{\boldsymbol{Z}_{1} \ldots \uplus \boldsymbol{Z}_{n} \uplus \boldsymbol{Z}^{u}=\boldsymbol{Z}} l\left(\boldsymbol{Z}^{u} \mid \boldsymbol{U}, \boldsymbol{s}\right) \\
& \times \prod_{i=1}^{n} t\left(\boldsymbol{Z}_{i} \mid \boldsymbol{X}_{i}, \boldsymbol{s}\right),
\end{aligned}
$$

where $\left|\boldsymbol{X}_{i}\right| \leq 1$ for $i=1, \ldots, n$. Here, $l\left(\boldsymbol{Z}^{u} \mid \boldsymbol{U}, \boldsymbol{s}\right)$ was defined in (12) and

$$
t\left(\boldsymbol{Z}_{i} \mid \boldsymbol{X}_{i}, \boldsymbol{s}\right)= \begin{cases}p_{D}(\boldsymbol{s}, \boldsymbol{x}) g(\boldsymbol{z} \mid \boldsymbol{s}, \boldsymbol{x}) & \boldsymbol{Z}_{i}=\{\boldsymbol{z}\}, \boldsymbol{X}_{i}=\{\boldsymbol{x}\}, \\ 1-p_{D}(\boldsymbol{s}, \boldsymbol{x}) & \boldsymbol{Z}_{i}=\emptyset, \boldsymbol{X}_{i}=\{\boldsymbol{x}\}, \\ 1 & \boldsymbol{Z}_{i}=\emptyset, \boldsymbol{X}_{i}=\emptyset, \\ 0 & \text { otherwise. }\end{cases}
$$

\section{Communication}

We assume that every vehicle is able to communicate all obtained measurements (V2F and GNSS) with the RSU instantaneously and without errors. This implies that at any time step $t$ the number of vehicles communicating with the RSU can vary. The incorporation of a realistic vehicle-toinfrastructure (V2I) channel model and its performance impact is outside the scope of this work.

\section{Proposed FILTER}

In this section, we formulate the proposed Bayesian filter with uncertain vehicle (sensor) state. For complexity reasons, we aim for a recursive formulation with a factorized joint density over the vehicle and feature states.

\section{A. Prior Joint State Density}

The vehicle state PDF at time step $t-1$ is indicated by subscript '-', i.e., $p_{-}(s)$, the PDF predicted to the current time step $t$ (before updating by a measurement) is indicated by subscript ' + ', i.e., $p_{+}(s)$, and the posterior PDF is stated without subscript. Similar notation holds w.r.t. the feature RFS density. With a single vehicle, the prior joint vehicle-feature density is of form

$$
f_{-}(s, \boldsymbol{X})=p_{-}(s) f_{-}(\boldsymbol{X}),
$$

where $p_{-}(s)$ is the prior PDF on the vehicle state, and $f_{-}(\boldsymbol{X})$ is the prior PMBM density. The latter density can be written in terms of a PPP density of unknown features $f_{-}^{u}\left(\boldsymbol{X}^{u}\right)$, i.e., features which are hypothesized to exist but have never been detected [12, Def. I], and the prior MBM RFS density of detected features, as [5, p. 484], [12]

$$
f_{-}(\boldsymbol{X}) \propto \sum_{\boldsymbol{X}^{u} \uplus \boldsymbol{X}_{1} \uplus \ldots \uplus \boldsymbol{X}_{n}=\boldsymbol{X}} f_{-}^{u}\left(\boldsymbol{X}^{u}\right) \sum_{j \in \mathbb{J}} \prod_{i \in \mathbb{I}_{j}} w_{j i} f_{j i-}\left(\boldsymbol{X}_{i}\right),
$$

where the $w_{j i}$ and $f_{j i-}(\cdot)$ are the weight and Bernoulli density of potentially detected feature $i$ under global hypothesis $j$. Note that the weight of a global hypothesis $j$ is proportional to the single hypothesis weights by $\prod_{i \in \mathbb{I}_{j}} w_{j i}$. In (17), the PPP density of unknown features is

$$
f_{-}^{u}\left(\boldsymbol{X}^{u}\right)=e^{-\left\langle D_{-}^{u}, 1\right\rangle} \prod_{\boldsymbol{x} \in \boldsymbol{X}^{u}} D_{-}^{u}(\boldsymbol{x})
$$

where $D_{-}^{u}(\boldsymbol{x})$ is the intensity of unknown features.

\section{B. Prediction Step}

Due to the independent mobility of vehicle and features, the predicted joint vehicle-feature density is

$$
f_{+}(s, \boldsymbol{X})=p_{+}(s) f_{+}(\boldsymbol{X}),
$$

where $p_{+}(s)$ is given by the Chapman-Kolmogorov equation $p_{+}(\boldsymbol{s})=\int p\left(\boldsymbol{s} \mid \boldsymbol{s}^{\prime}\right) p_{-}\left(\boldsymbol{s}^{\prime}\right) \mathrm{d} \boldsymbol{s}^{\prime}$, where $p\left(\boldsymbol{s} \mid \boldsymbol{s}^{\prime}\right)$ is the state transition PDF and $p_{-}\left(s^{\prime}\right)$ is the prior PDF [36]. Similarly, the predicted feature state PMB density is calculated by [5, Ch. 14.3]

$$
f_{+}(\boldsymbol{X})=\int f\left(\boldsymbol{X} \mid \boldsymbol{X}^{\prime}\right) f_{-}\left(\boldsymbol{X}^{\prime}\right) \delta \boldsymbol{X}^{\prime},
$$

where $f\left(\boldsymbol{X} \mid \boldsymbol{X}^{\prime}\right)$ is the transition RFS density, and $f_{-}\left(\boldsymbol{X}^{\prime}\right)$ is the prior PMB density. It can be shown that (20) is a PMB density where the predicted intensity of unknown features $D_{+}^{u}(\boldsymbol{x})$ is given by $[12$, Thm. I]

$$
D_{+}^{u}(\boldsymbol{x})=D^{b}(\boldsymbol{x})+\int p_{\mathrm{S}}\left(\boldsymbol{x}^{\prime}\right) p\left(\boldsymbol{x} \mid \boldsymbol{x}^{\prime}\right) D_{-}^{u}\left(\boldsymbol{x}^{\prime}\right) \mathrm{d} \boldsymbol{x}^{\prime} .
$$

Here, the (known) birth intensity is denoted $D^{b}(\boldsymbol{x}), p\left(\boldsymbol{x} \mid \boldsymbol{x}^{\prime}\right)$ denotes the feature transition PDF, and the feature survival probability is denoted $p_{\mathrm{S}}\left(\boldsymbol{x}^{\prime}\right)$. The Bernoulli components of the MBM are updated as follows [12, Eqn. (40)]:

$$
\begin{aligned}
p_{j i+}(\boldsymbol{x}) & =\int p_{\mathrm{S}}\left(\boldsymbol{x}^{\prime}\right) p\left(\boldsymbol{x} \mid \boldsymbol{x}^{\prime}\right) p_{j i-}\left(\boldsymbol{x}^{\prime}\right) \mathrm{d} \boldsymbol{x}^{\prime} \\
r_{j i+} & =r_{j i-} \int p_{\mathrm{S}}\left(\boldsymbol{x}^{\prime}\right) p_{j i-}\left(\boldsymbol{x}^{\prime}\right) \mathrm{d} \boldsymbol{x}^{\prime}
\end{aligned}
$$

where $p_{j i-}\left(\boldsymbol{x}^{\prime}\right)$ denotes the prior PDF of the $j i$-th Bernoulli component.

\section{Measurement Update Step}

Updating the joint vehicle-feature density (19) by any of the two types of different measurements, GNSS and V2F measurements, involves the application of Bayes' theorem. In the following, we describe the update calculations using the different type of measurements. 
1) Update with vehicle state measurement: Let $z_{\mathrm{G}}$ be a measurement related to the vehicle state $s$ through $p\left(\boldsymbol{z}_{\mathrm{G}} \mid \boldsymbol{s}\right)$. Given a predicted vehicle-feature density (19), by Bayes' theorem the updated density is

$$
f\left(\boldsymbol{s}, \boldsymbol{X} \mid \boldsymbol{z}_{\mathrm{G}}\right)=\frac{p\left(\boldsymbol{z}_{\mathrm{G}} \mid \boldsymbol{s}\right) p_{+}(\boldsymbol{s})}{\int p\left(\boldsymbol{z}_{\mathrm{G}} \mid \boldsymbol{s}^{\prime}\right) p_{+}\left(\boldsymbol{s}^{\prime}\right) \mathrm{d} \boldsymbol{s}^{\prime}} f_{+}(\boldsymbol{X}) .
$$

In other words, the vehicle state density is updated with the measurement $\boldsymbol{z}_{\mathrm{G}}$, the feature set density is unaffected by the update, and the independent form is retained (c.f. (16)).

2) Update with cluttered set of feature measurements: Let $Z$ be the V2F measurement subject to the model defined in Section III-C. Furthermore, we assume the probability of detection to be state-independent, i.e., $p_{\mathrm{D}}(\boldsymbol{s}, \boldsymbol{x})=p_{\mathrm{D}}$.

With the help of Bayes' rule, the updated joint vehiclefeature density is

$$
f(\boldsymbol{s}, \boldsymbol{X} \mid \boldsymbol{Z}) \propto p_{+}(\boldsymbol{s}) f_{+}(\boldsymbol{X}) l(\boldsymbol{Z} \mid \boldsymbol{s}, \boldsymbol{X}) .
$$

Due to the dependency of the V2F measurements on $\boldsymbol{X}$ and $s$, the updated posterior density (25) cannot easily be decomposed into the independent form of the prior joint density (16). In order to enable joint vehicle-feature state tracking with: (i) low complexity; and (ii) standard MTT frameworks such as, e.g., [12], we calculate (25) approximately. With the help of Lemma 1 the joint vehicle-feature density is approximated as

$$
f(\boldsymbol{s}, \boldsymbol{X} \mid \boldsymbol{Z}) \approx p(\boldsymbol{s} \mid \boldsymbol{Z}) f(\boldsymbol{X} \mid \boldsymbol{Z}),
$$

where

$$
\begin{aligned}
p(\boldsymbol{s} \mid \boldsymbol{Z}) & \propto \int p_{+}(\boldsymbol{s}) f_{+}(\boldsymbol{X}) l(\boldsymbol{Z} \mid \boldsymbol{s}, \boldsymbol{X}) \delta \boldsymbol{X}, \\
f(\boldsymbol{X} \mid \boldsymbol{Z}) & \propto \int p_{+}(\boldsymbol{s}) f_{+}(\boldsymbol{X}) l(\boldsymbol{Z} \mid \boldsymbol{s}, \boldsymbol{X}) \mathrm{d} \boldsymbol{s} .
\end{aligned}
$$

In this way, the independent structure of the vehicle and feature density (16) is retained. Note that it has been observed in the SLAM context that a factorization such as (26) can generate optimistic estimates about the state uncertainty [18], [37].

An alternative to the approximation (26) is to perform a Rao-Blackwellization as done in SLAM [18], [21]. Although more accurate, the extension of the multi-vehicle scenario is not apparent and opponent to a low complexity implementation we seek.

Now, only computation of the marginal posteriors remains. This is derived in the next two subsections. For convenience, let

$$
\mathcal{X}_{\boldsymbol{Z}}(\boldsymbol{z})= \begin{cases}0 & \boldsymbol{z} \notin \boldsymbol{Z}, \\ 1 & \boldsymbol{z} \in \boldsymbol{Z},\end{cases}
$$

and

$$
\delta_{\emptyset}(\boldsymbol{Y})= \begin{cases}0 & \boldsymbol{Y} \neq \emptyset \\ 1 & \boldsymbol{Y}=\emptyset .\end{cases}
$$

\section{Marginal Feature Set Posterior}

The feature posterior $f(\boldsymbol{X} \mid \boldsymbol{Z})$ of (26) is given by the following Theorem.
Theorem 3: The feature posterior for measurement set $\boldsymbol{Z}=$ $\left\{\boldsymbol{z}_{1}, \ldots, \boldsymbol{z}_{m}\right\}$ can be approximated by a PMBM with density

$$
\begin{aligned}
f(\boldsymbol{X} \mid \boldsymbol{Z}) & \stackrel{\sum_{\boldsymbol{Y} \uplus \boldsymbol{X}_{1} \ldots \uplus \boldsymbol{X}_{n} \uplus \boldsymbol{X}_{1} \ldots \uplus \boldsymbol{X}_{m}=\boldsymbol{X}} f(\boldsymbol{Y}) \sum_{j}}{ } \\
& \times \sum_{\boldsymbol{Z}_{1} \ldots \uplus \boldsymbol{Z}_{n} \uplus \boldsymbol{Z}^{u}=\boldsymbol{Z}} \prod_{i=1}^{m}\left[\mathcal{X}_{\boldsymbol{Z}^{u}}\left(\boldsymbol{z}_{i}\right) \rho\left(\boldsymbol{z}_{i}\right) f\left(\boldsymbol{X}_{i} \mid \boldsymbol{z}_{i}\right)\right. \\
& \left.+\left(1-\mathcal{X}_{\boldsymbol{Z}^{u}}\left(\boldsymbol{z}_{i}\right)\right) \delta_{\emptyset}\left(\boldsymbol{X}_{i}\right)\right] \\
& \times \prod_{i=1}^{n} w_{j i} \rho_{j i}\left(\boldsymbol{Z}_{i}\right) f_{j i}\left(\boldsymbol{X}_{i} \mid \boldsymbol{Z}_{i}\right),
\end{aligned}
$$

where $\underset{\sim}{\sim}$ means approximately equal to. Here, $f(\boldsymbol{Y})$ represents the PPP component, and the functional forms of the densities $f\left(\boldsymbol{X}_{i} \mid \boldsymbol{z}_{i}\right)$ and $f_{j i}\left(\boldsymbol{X}_{i} \mid \boldsymbol{Z}_{i}\right)$, as well as the constants $\rho\left(\boldsymbol{z}_{i}\right)$, $\rho_{j i}\left(\boldsymbol{Z}_{i}\right)$ are provided in Appendix ??.

Proof: See Appendix ??.

The feature posterior (31) consists of undetected features $(\boldsymbol{Y})$, hypotheses for newly detected features $\left(\boldsymbol{X}_{i}\right)$ and for updating existing features $\left(\boldsymbol{X}_{i}\right)$. This structure is of the same form $^{2}$ as for a known vehicle (sensor) state, as in [13], and is thus amenable for using a standard PMBM filter implementation [12]. The weights of newly detected features are considered in the product over $m$ components (i.e., one new feature per measurement). A previously detected feature has an updated weight $w_{j i} \rho_{j i}\left(\boldsymbol{Z}_{i}\right)$ consisting of the previous single hypothesis weight and, depending on whether $\boldsymbol{Z}_{i}$ contains a measurement, the hypotheses for a detection or a miss of the feature.

Note that (31) is only approximately $f(\boldsymbol{X} \mid \boldsymbol{Z})$ due to the approximations performed in the derivations. Therefore, the weights are also only approximate weights for $f(\boldsymbol{X} \mid \boldsymbol{Z})$.

\section{E. Marginal Vehicle Posterior}

We now proceed with the vehicle state posterior, which is stated in the following Theorem.

Theorem 4: The vehicle state posterior for measurement set $Z$ is

$$
\begin{aligned}
p(\boldsymbol{s} \mid \boldsymbol{Z}) & \propto \sum_{j} \sum_{\boldsymbol{Z}_{1} \ldots \uplus \boldsymbol{Z}_{n} \uplus \boldsymbol{Z}^{u}=\boldsymbol{Z}} \prod_{\boldsymbol{z}_{i} \in \boldsymbol{Z}^{u}} \mathcal{X}_{\boldsymbol{Z}^{u}}\left(\boldsymbol{z}_{i}\right) \rho\left(\boldsymbol{z}_{i}\right) \\
& \times \prod_{i=1}^{n} w_{j i} \rho_{j i}\left(\boldsymbol{Z}_{i}\right) q_{j}\left(\boldsymbol{s} \mid \boldsymbol{Z}_{1}, \ldots, \boldsymbol{Z}_{n}, \boldsymbol{Z}^{u}\right)
\end{aligned}
$$

where $q_{j}\left(\boldsymbol{s} \mid \boldsymbol{Z}_{1}, \ldots, \boldsymbol{Z}_{n}, \boldsymbol{Z}^{u}\right)$ is a properly normalized density defined in Appendix ??.

Proof: See Appendix ??.

From the vehicle posterior (32), we observe that the single hypothesis weights $\rho\left(\boldsymbol{z}_{i}\right)$ and $w_{j i} \rho_{j i}\left(\boldsymbol{Z}_{i}\right)$ of each term are the same as in the feature posterior (31), only the order of integration over the vehicle state $s$ and the single feature state $\boldsymbol{x}$ is exchanged. Hence, both posteriors use the same approximate single hypothesis weights. Furthermore, we have obtained that a global hypothesis is approximately proportional to the product of the approximate single hypothesis weights.

\footnotetext{
${ }^{2}$ For the linear Gaussian case, the vehicle state uncertainty is essentially mapped onto the V2F measurement uncertainty, which can be seen as an increased time-varying measurement variance.
} 


\section{F. Implementation Aspects}

Here, we discuss approximations for a practical implementation to perform the sensor state V2F measurement update when the probability of existence of detected features is high. Furthermore, we discuss the approximation of the joint DA and the reduction of the feature posterior density to contain only a single global hypothesis. Using this approximation, the complexity of the proposed filter is briefly discussed.

1) Certain Feature Information: The spatial PDF of the PPP modeling the undetected features needs to cover the whole space where new features appear. Due to this, a newly detected feature does not provide certain information to update the vehicle state and may be neglected in the vehicle state update. Furthermore, one can approximate the vehicle state update by considering only previously detected features with a high existing probability (c.f. Sec. 2). When detection probability is high, (32) can be approximated by

$$
\begin{aligned}
p(\boldsymbol{s} \mid \boldsymbol{Z}) & \stackrel{\sim}{\sim} \sum_{j} \sum_{\boldsymbol{Z}_{1} \ldots \uplus \boldsymbol{Z}_{n} \uplus \boldsymbol{Z}^{u}=\boldsymbol{Z}} \\
& \times\left(\prod_{i=1}^{n} w_{j i} \rho_{j i}\left(\boldsymbol{Z}_{i}\right)\right) q_{j}\left(\boldsymbol{s} \mid \boldsymbol{Z}_{1}, \ldots, \boldsymbol{Z}_{n}, \boldsymbol{Z}^{u}\right),
\end{aligned}
$$

where

$$
\begin{aligned}
& q_{j}\left(\boldsymbol{s} \mid \boldsymbol{Z}_{1}, \ldots, \boldsymbol{Z}_{n}, \boldsymbol{Z}^{u}\right) \\
& \propto p_{+}(\boldsymbol{s}) \prod_{i=1}^{n}\left(1-\delta_{\emptyset}\left(\boldsymbol{Z}_{i}\right)\right) \int g\left(\boldsymbol{z}_{i} \mid \boldsymbol{x}_{i}, \boldsymbol{s}\right) p_{j i}\left(\boldsymbol{x}_{i}\right) \mathrm{d} \boldsymbol{x}_{i} .
\end{aligned}
$$

2) Marginal Association: For the feature update, the global hypothesis weights are proportional to $\prod_{\boldsymbol{z}_{i} \in \boldsymbol{Z}^{u}} \rho\left(\boldsymbol{z}_{i}\right) \prod_{i=1}^{n} w_{j i} \rho_{j i}\left(\boldsymbol{Z}_{i}\right)$. For the vehicle state update, the global hypothesis weight is proportional to $\left.\prod_{i=1}^{n} w_{j i} \rho_{j i}\left(\boldsymbol{Z}_{i}\right)\right)$. A global hypothesis over all features can be approximated by the product of marginal association weights using the FG approach of [38].

For the feature state update, the TOMB/P filter [12] permits the reduction of the posterior PMBM density to a PMB density containing only a single global hypothesis (so that the summation over $j$ disappears in the next time step).

For the vehicle state update, each likelihood term is weighted w.r.t. the single hypothesis. From the feature state update, the obtained marginal data association weights for the feature update can be reused in the vehicle state update, since they use the same weights for the same hypotheses. The weighted likelihoods times the prior vehicle state is then approximately proportional to the vehicle state posterior conditioned on the measurement set $\boldsymbol{Z}$.

3) Complexity: For Gaussian linear models, a GNSS measurement update (c.f. (24)) has same complexity as a Kalman filter update. Using the marginal association proposed by the TOMB/P filter, the update of the feature state density by $\mathrm{V} 2 \mathrm{~F}$ measurements has comparable complexity as an update step of the TOMB/P filter with the added complexity of the marginalization over the sensor state (c.f. Appendix ??). For the vehicle state update with the $\mathrm{V} 2 \mathrm{~F}$ measurements we have the same number of hypotheses and hence similar complexity.

\section{G. Multi-Vehicle Generalization of Proposed Filter}

Up to this point, we discussed joint vehicle-feature state tracking using a single vehicle, where GNSS and V2F measurements are incorporated. To achieve feature tracking as described in Section III-A, where a GNSS and a V2F sensor is mounted on each vehicle, we have to consider the multivehicle/multisensor case, where each vehicle is equipped with a GNSS and a V2F sensor. Since GNSS measurements are straightforward to deal with (they can be applied prior to the $\mathrm{V} 2 \mathrm{~F}$ measurements), we focus only on V2F measurements, considering a two-vehicle case. There are different approaches to handle the multi-vehicle setting, where we highlight two of them next.

1) Parallel Approach: Given time-synchronized measurements from 2 vehicles (with measurements $Z_{1}$ collected by vehicle 1 and $Z_{2}$ collected by vehicle 2 , respectively), the joint posterior can be approximated by

$$
\begin{aligned}
& f\left(\boldsymbol{s}_{1}, \boldsymbol{s}_{2}, \boldsymbol{X} \mid \boldsymbol{Z}_{1}, \boldsymbol{Z}_{2}\right) \\
& \approx p\left(\boldsymbol{s}_{1}, \boldsymbol{s}_{2} \mid \boldsymbol{Z}_{1}, \boldsymbol{Z}_{2}\right) f\left(\boldsymbol{X} \mid \boldsymbol{Z}_{1}, \boldsymbol{Z}_{2}\right)
\end{aligned}
$$

similar to the approximation (26). Note that the set of global hypotheses is now the Cartesian product of the individual vehicle's set of hypotheses. Several different approaches can be employed to tackle this DA problem in a tractable manner. For instance, by employing sequential sensor-by-sensor measurement updates (also called iterator-corrector method) [5], or by partition of the measurement set into subsets associated with the Bernoulli components [39], or by performing variational inference [40], or by solving the DA in parallel on a sensorby-sensor basis [16]. Note that in a system where sensors are spatially distributed (c.f. Sec. III-A) synchronization between sensors is involved and a sequential measurement update procedure may be used instead.

2) Sequential Approach: Here, we employ the sequential measurement update strategy together with the TOMB/P algorithm (c.f. Section IV-C). Note that in a real system sensors among different vehicles are difficult to synchronize and then this approach can be used. The update is first performed with respect to the first vehicle (c.f. Sec. IV-D and Sec. IV-E):

$$
\begin{aligned}
& f\left(\boldsymbol{s}_{1}, \boldsymbol{X} \mid \boldsymbol{Z}_{1}\right) \stackrel{\propto}{\sim} p_{+}\left(\boldsymbol{s}_{1}\right) l\left(\boldsymbol{Z}_{1} \mid \boldsymbol{s}_{1}, \boldsymbol{X}\right) f_{+}(\boldsymbol{X}) \delta \boldsymbol{X} \\
& \times \int p_{+}\left(\boldsymbol{s}_{1}\right) l\left(\boldsymbol{Z}_{1} \mid \boldsymbol{s}_{1}, \boldsymbol{X}\right) f_{+}(\boldsymbol{X}) \mathrm{d} \boldsymbol{s}_{1} \\
& =p\left(\boldsymbol{s}_{1} \mid \boldsymbol{Z}_{1}\right) f\left(\boldsymbol{X} \mid \boldsymbol{Z}_{1}\right) .
\end{aligned}
$$

Then, the density $f\left(\boldsymbol{X} \mid \boldsymbol{Z}_{1}\right)$ is used as a prior when performing the update with respect to the second vehicle:

$$
\begin{aligned}
& f\left(\boldsymbol{s}_{2}, \boldsymbol{X} \mid \boldsymbol{Z}_{1}, \boldsymbol{Z}_{2}\right) \stackrel{\propto}{\sim} p_{+}\left(\boldsymbol{s}_{2}\right) l\left(\boldsymbol{Z}_{2} \mid \boldsymbol{s}_{2}, \boldsymbol{X}\right) f\left(\boldsymbol{X} \mid \boldsymbol{Z}_{1}\right) \delta \boldsymbol{X} \\
& \times \int p_{+}\left(\boldsymbol{s}_{2}\right) l\left(\boldsymbol{Z}_{2} \mid \boldsymbol{s}_{2}, \boldsymbol{X}\right) f\left(\boldsymbol{X} \mid \boldsymbol{Z}_{1}\right) \mathrm{d} \boldsymbol{s}_{2} \\
& =p\left(\boldsymbol{s}_{2} \mid \boldsymbol{Z}_{1}, \boldsymbol{Z}_{2}\right) f\left(\boldsymbol{X} \mid \boldsymbol{Z}_{1}, \boldsymbol{Z}_{2}\right) .
\end{aligned}
$$

Using this method, subsequent vehicles will benefit from updated vehicle and feature information of preceding vehicles. Note that contrary to (35), the vehicle states are approximated here as being independent of each other. In our application 


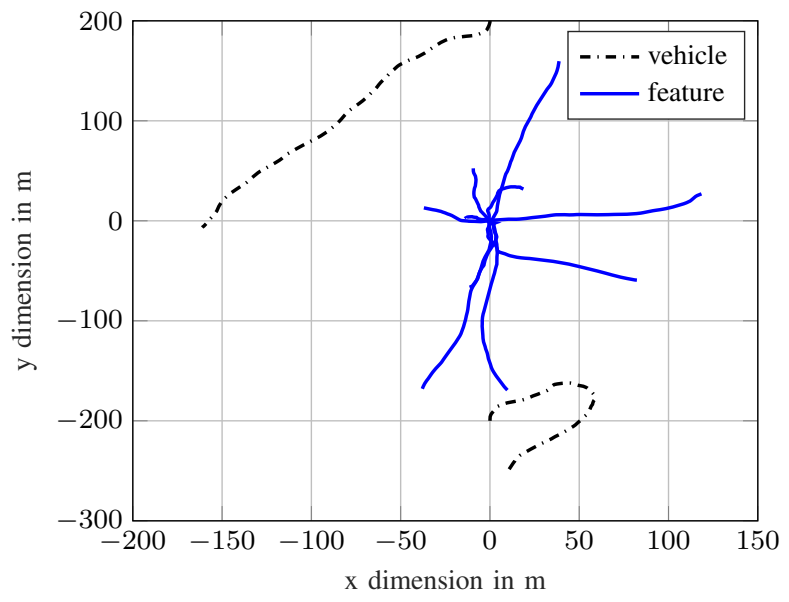

Fig. 2. One realization of two vehicle and five feature trajectories for the experiment with synthetic data.

example (c.f. Section III-A), this means that an update of the joint vehicle-feature density, with measurements from a well-localized vehicle (certain vehicle state), results in an improvement of feature tracking performance when prior information on the features is low. An update of the joint vehiclefeature density, with measurements from a poorly localized vehicle (uncertain vehicle state), permits the reduction of the uncertainty of its own vehicle state when prior information on the features is high.

\section{Results With Synthetic Data}

We consider a scenario similar to the one outlined in Fig. 1, where we apply the proposed multisensor-multifeature state tracking filter presented in Section IV.

\section{A. Setup}

The state of vehicle $v \in \mathcal{V}$ at time step $t$ is $\boldsymbol{s}_{v, t}=$ $\left[\boldsymbol{p}_{s, t}^{\top}, \dot{\boldsymbol{p}}_{v, t}^{\top}\right]^{\top}$ with position $\boldsymbol{p}_{v, t} \in \mathbb{R}^{2}$ and velocity $\dot{\boldsymbol{p}}_{v, t} \in \mathbb{R}^{2}$. We use a linear constant velocity $(\mathrm{CV})$ model to model vehicle dynamics, where

$$
\boldsymbol{s}_{s, t}=\boldsymbol{A} \boldsymbol{s}_{s, t-1}+\boldsymbol{w}_{s, t}
$$

with state-transition matrix

$$
\boldsymbol{A}=\left[\begin{array}{cc}
1 & T_{s} \\
0 & 1
\end{array}\right] \otimes \boldsymbol{I}_{2},
$$

where $T_{s}=0.5 \mathrm{~s}$ is the sampling time. Above, $\boldsymbol{w}_{s, t} \sim$ $\mathcal{N}(0, \boldsymbol{W})$ denotes the IID process noise with

$$
\boldsymbol{W}=r\left[\begin{array}{cc}
T_{s}^{3} / 3 & T_{s}^{2} / 2 \\
T_{s}^{2} / 2 & T_{s}
\end{array}\right] \otimes \boldsymbol{I}_{2},
$$

where $r=0.05 \mathrm{~m}^{2}$. Above, $\otimes$ denotes the Kronecker product. We set the initial state of vehicle 1 to $s_{1,0}=[0,200,0,-2]^{\top}$ and to $s_{2,0}=[0,-200,0,2]^{\top}$ for vehicle 2 , respectively. The state of feature $k$ at time $t$, denoted $\boldsymbol{x}_{k, t} \in \mathbb{R}^{4}$, is comprised of Cartesian position and velocity, similar to the vehicle state $s_{v, t}$. There are at most five features present, if not noted otherwise. Furthermore, feature dynamics follow the
CV model with the same parameters used for the vehicles. To generate a challenging scenario for DA, we initialize the feature states $\boldsymbol{x}_{k, t} \sim \mathcal{N}\left(0,0.25 \boldsymbol{I}_{4}\right)$ at time step $t=175$ for all features $k \in \mathcal{K}$ and run the $\mathrm{CV}$ model forward and backward in time similar to [12, Sec. VI]. The first feature enters the scene after $t=0$, the second after $t=20$ and so on. Once present, features stay alive for the remaining simulation time. A realization of vehicle and feature trajectories is shown in Fig. 2.

For the GNSS measurements, we use the linear measurement model

$$
\boldsymbol{z}_{\mathrm{G}, t}=\boldsymbol{H}_{\mathrm{G}} \boldsymbol{s}_{t}+\boldsymbol{r}_{t},
$$

where the observation matrix is $\boldsymbol{H}_{\mathrm{G}}=\left[\begin{array}{ll}1 & 0\end{array}\right] \otimes \boldsymbol{I}_{2}$ and $\boldsymbol{r}_{t} \sim$ $\mathcal{N}(0, \boldsymbol{R})$ denotes the measurement noise with $\boldsymbol{R}=\sigma_{\mathrm{G}}^{2} \boldsymbol{I}_{2}$. For vehicle 1, we assume it has low location uncertainty using Real Time Kinematic (RTK) with $\sigma_{\mathrm{G}}^{2}=5.76 \cdot 10^{-4} \mathrm{~m}^{2}$ and for vehicle 2 high location uncertainty using Standard Positioning System (SPS) with $\sigma_{\mathrm{G}}^{2}=12.96 \mathrm{~m}^{2}$, corresponding to a vehicle with high quality GNSS receiver and one with low quality GNSS receiver ${ }^{3}$. In the single sensor case, only vehicle 1 is present, and in the multisensor case both vehicles are present, if not noted otherwise.

For the V2F measurements, we use the linear measurement model

$$
\boldsymbol{z}_{t}=\boldsymbol{H}_{1} \boldsymbol{s}_{t}+\boldsymbol{H}_{2} \boldsymbol{x}_{t}+\boldsymbol{q}_{t}
$$

with $\boldsymbol{H}_{1} \triangleq \boldsymbol{H}_{\mathrm{G}}$ and $\boldsymbol{H}_{2} \triangleq-\boldsymbol{H}_{\mathrm{G}}$, and $\boldsymbol{q}_{t} \sim$ $\mathcal{N}(0, \boldsymbol{Q})$ with measurement noise covariance matrix $\boldsymbol{Q}=$ $\sigma_{\mathrm{V} 2 \mathrm{~F}}^{2} \boldsymbol{I}_{2}$. Following [12], we set the initial unknown feature intensity to $D_{-}^{u}\left(\boldsymbol{x}_{k, t}\right)=10 \mathcal{N}(\mathbf{0}, \boldsymbol{P})$, where $\boldsymbol{P}=$ $\operatorname{diag}\left(\left[100^{2}, 100^{2}, 1,1\right]^{\top}\right)$ to cover the ranges of interest of the feature state. The feature birth intensity is set to $D^{b}\left(\boldsymbol{x}_{k, t}\right)=$ $0.05 \mathcal{N}(\mathbf{0}, \boldsymbol{P})$, the average number of false alarms per scan to $\lambda_{c}=10$, with uniform spatial distribution on $\left[-r_{\max }, r_{\max }\right]$ with parameter $r_{\max }=500 \mathrm{~m}$. Furthermore, the probability of survival is $p_{\mathrm{S}}=0.7$ and the probability of detection is $p_{\mathrm{D}}\left(\boldsymbol{s}_{v, t}, \boldsymbol{x}_{k, t}\right) \triangleq p_{\mathrm{D}}=0.9$. To assess feature tracking performance, we use the optimal sub-pattern assignment (OSPA) metric with cut-off parameter $c=20$ and order $p=2$ [43]. The filter tracking performance for the vehicle state at time step $t$ is assessed in terms of the position estimation error $e_{t}=\left\|\boldsymbol{p}_{t, \text { true }}-\hat{\boldsymbol{p}}_{t}\right\|_{2}$, where $\boldsymbol{p}_{t, \text { true }}$ is the true vehicle position and $\hat{\boldsymbol{p}}_{t}$ the mean estimate of the filter.

We analyze the proposed MTT filter w.r.t. feature tracking performance with sensor update from the tracked features to the sensor (proposed, sensor update), and without sensor update (proposed, no sensor update). For comparison, results are shown using the TOMB/P filter ignoring sensor state uncertainty [12], i.e., using the GNSS measurement as sensor state (TOMB/P I, no sensor update), and the TOMB/P filter ignoring sensor state uncertainty, but increasing the V2F variance by the GNSS measurement variance (TOMB/P II, no sensor update), which is possible in the considered

\footnotetext{
${ }^{3}$ According to [41] and [42], the $\mathrm{x} / \mathrm{y}$ position accuracy of the GNSS receiver RT3000 from OXTS is $\sigma_{\mathrm{G}}^{2}=12.96 \mathrm{~m}^{2}$ for SPS, $\sigma_{\mathrm{G}}^{2}=2.0736 \mathrm{~m}^{2}$ for Satellite-Based Augmentation System (SBAS), $\sigma_{\mathrm{G}}^{2} \stackrel{\sigma_{\mathrm{G}}}{=} 0.9216 \mathrm{~m}^{2}$ for Differential Global Positioning System (DGPS), and $\sigma_{\mathrm{G}}^{2}=5.76 \cdot 10^{-4} \mathrm{~m}^{2}$ for RTK.
} 


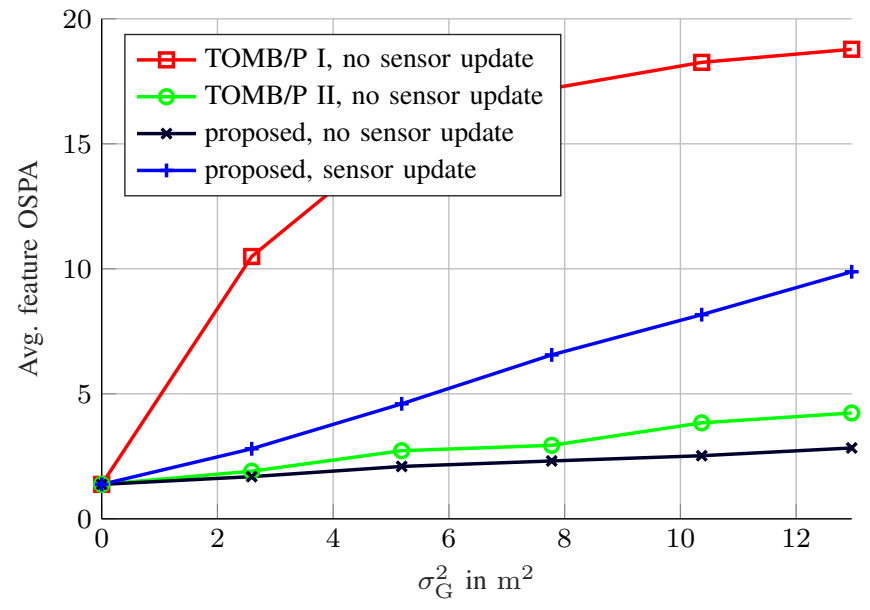

Fig. 3. Average feature OSPA for different values of GNSS measurement variance $\sigma_{\mathrm{G}}^{2}$ using the proposed MTT filter with sensor update from the tracked features to the sensor (proposed, sensor update) and without sensor update (proposed, no sensor update). For comparison, results using the TOMB/P filter ignoring sensor state uncertainty (TOMB/P I, no sensor update) and by increasing the V2F noise variance by the GNSS variance (TOMB/P II, no sensor update) are shown.

linear measurement scenario. As a benchmark for vehicle localization performance, results from a centralized Kalman filter (KF) are plotted as well, where measurement-to-feature DA is known and where the augmented state vector contains all vehicle and all feature states. This is denoted genie method. Furthermore, tracking performance using a local KF is plotted. The local KF performs filtering separately on the individual vehicle state using only GNSS measurements and does not estimate feature states. Note, the performance of the local KF can be considered as the worst-case performance on vehicle state estimation, since V2F measurements are not considered at all.

\section{B. Discussion}

First, we discuss the impact of an uncertain vehicle state on feature tracking performance using a single vehicle and multiple features. After that, we consider the multisensormultifeature case from Section IV-G.

1) Impact of uncertain vehicle state on feature tracking performance: For one simulation run, the features and vehicle trajectories are outlined in Fig. 2. For the case the GNSS measurement variance is very small w.r.t. the $\mathrm{V} 2 \mathrm{~F}$ measurement variance, e.g., $\sigma_{\mathrm{G}}^{2}=5.76 \cdot 10^{-4} \mathrm{~m}^{2}$ corresponding to RTK and $\sigma_{\mathrm{V} 2 \mathrm{~F}}^{2}=0.42 \mathrm{~m}^{2}$, the feature tracking performance of the proposed filter is comparable with the TOMB/P filter with known sensor state. Hence, we will not focus on this case and refer the reader to [12] for performance results regarding the $\mathrm{TOMB} / \mathrm{P}$ filter.

In Fig. 3, the feature state OSPA average of one simulation run of 351 time steps averaged over 50 Monte-Carlo (MC) trajectory realizations is plotted for different values of GNSS measurement variance $\sigma_{\mathrm{G}}^{2}$ for two variants of the proposed MTT filter as well as with the TOMB/P filter. For all filter variants, the increase of GNSS measurement variance leads to an increased vehicle state uncertainty with the effect of an

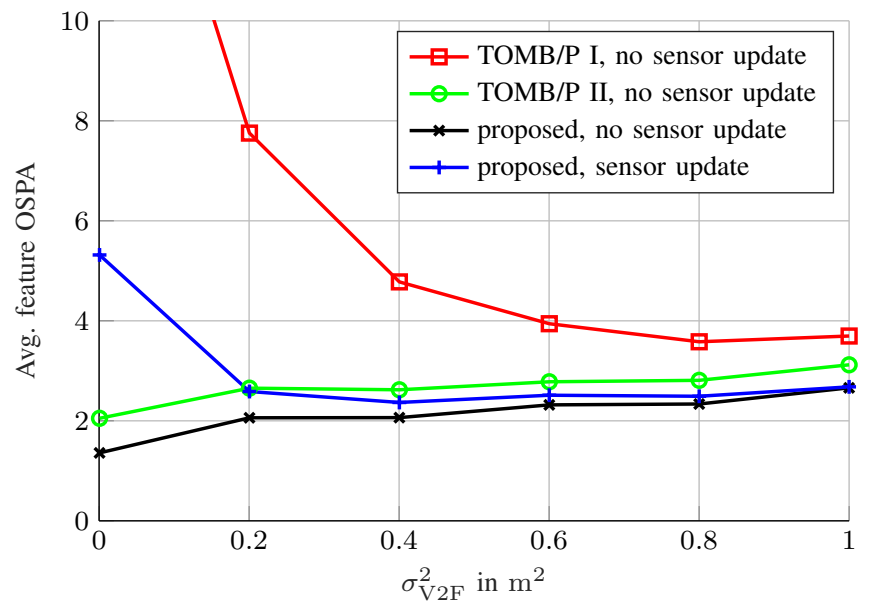

Fig. 4. Average feature OSPA for different values of $\mathrm{V} 2 \mathrm{~F}$ measurement variance $\sigma_{\mathrm{V} 2 \mathrm{~F}}^{2}$. The GNSS noise variance is set to $\sigma_{\mathrm{G}}^{2}=0.9216 \mathrm{~m}^{2}$.

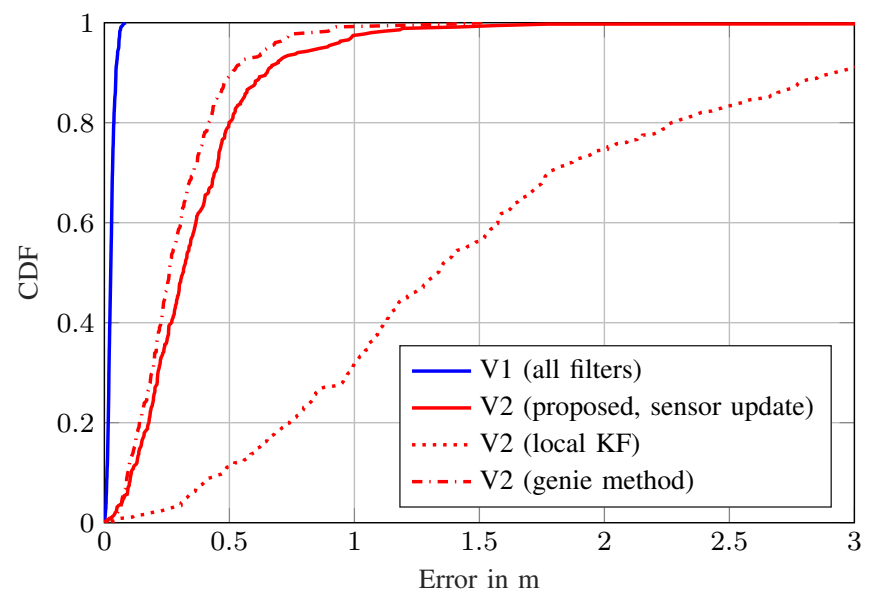

Fig. 5. CDF plot of vehicle position estimation error.

increase of the average feature OSPA. This OSPA increase consists of two components. First, an increased feature state estimation error due to a higher value of $\sigma_{\mathrm{G}}^{2}$. Second, this results in features appearing in the filter spatially close together w.r.t. the feature state measurement uncertainty $\left(\sigma_{\mathrm{G}}^{2}\right.$ and $\sigma_{\mathrm{V} 2 \mathrm{~F}}^{2}$ together) for a longer period of time around time step $t=175$. Hence, DA is more challenging with the effect of an increased feature OSPA in this regime.

From the figure, we observe that not considering the present vehicle state uncertainty leads to the worst feature tracking performance (TOMB/P I). Incorporating this uncertainty by increasing the $\mathrm{V} 2 \mathrm{~F}$ measurement variance improves feature tracking significantly. Modeling the present vehicle state uncertainty without artificially increasing the V2F measurement variance, as proposed in this paper, leads to a slightly better feature tracking performance (proposed, no sensor update). For the case the vehicle state is updated by the V2F measurement (proposed, sensor update), feature tracking performance deteriorates. The reason for this is that feature and vehicle state become correlated after the V2F measurement update, which is not modeled by the proposed MTT filter. This leads to the conclusion that the sensor should not use V2F measurements 
to update its own state if no other vehicles have updated the sensor state in the previous time step. With more vehicles, this effect of correlation will be diluted. Similar observations have been reported in [33].

In Fig. 4, the average feature state OSPA is plotted for different values of $\mathrm{V} 2 \mathrm{~F}$ measurement noise variance $\sigma_{\mathrm{V} 2 \mathrm{~F}}^{2}$ using the different filter variants. We observe that a higher V2F noise variance leads to an increased average feature OSPA value except for the TOMB/P filter which ignores sensor state uncertainty (TOMB/P I). In the former, this reduction comes from the fact that sensor state uncertainty is absorbed from the increased V2F noise variance. For all methods the single feature state estimation error increases and DA becomes more challenging. Since the sensor state uncertainty is negligible, all filter variants show the same performance.

2) Multisensor-multifeature tracking performance: Here, we limit the discussion on the two vehicle (sensor) case since it is sufficient to analyze the effect of updating the vehicle state using V2F measurements. In Fig. 5, the cumulative distribution function $(\mathrm{CDF})$ of the vehicle position estimation error is plotted for the two vehicle scenario outlined in Sec. V-A. We observe that for vehicle 1, which has low GNSS measurement noise, all three filter methods deliver a similar performance. The reason for this is that, due to the high accuracy of GNSS measurements, not a lot of information (to improve the vehicle state) is provided from feature tracking.

Moving our focus to vehicle 2, we observe that the error of the local KF is much higher compared to the central KF, which is caused by the high noise in the GNSS measurements. Due to the low estimation error of vehicle 1's state, there is relevant position information in the system, which can be transfered from vehicle 1 to vehicle 2 via the features utilizing the $\mathrm{V} 2 \mathrm{~F}$ measurements. In $80 \%$ of all cases, the estimation error of vehicle 2 is below $0.5 \mathrm{~m}$ using the proposed filter, compared to $2.3 \mathrm{~m}$ using the local $\mathrm{KF}$.

Despite this great improvement of the proposed filter over the local KF, it does not achieve the performance of the central $\mathrm{KF}$ (genie method), where the error is around $0.4 \mathrm{~m}$. The reason for the difference is that the central KF has knowledge of the correct DA, knows the true number of features present, and ignores clutter V2F measurements. Furthermore, it tracks any present correlations between features and vehicles not modeled by the proposed filter. The proposed filter needs to infer the measurement-to-feature DA, estimate the number of features currently present, and needs to appropriately handle clutter in the V2F measurement set $Z$.

\section{RESUlts With EXPERIMENTAL DATA}

\section{A. Experiment Description}

Measurement data was recorded using the COPPLAR project test vehicle, a Volvo XC90 equipped with different kinds of automotive sensors. If not stated otherwise, the filter parameters are the same as described in Section V-A. The GNSS sensor is a high-precision Applanix POSLV, and the $\mathrm{V} 2 \mathrm{~F}$ sensor is a onboard stereo vision camera from Autoliv looking in the car driving direction, which provides detections of objects w.r.t. the sensor coordinate frame. Measurements from both sensors arrive time-stamped, but are not synchronized. In order to obtain synchronized measurements, we linearly interpolated measurements from each sensor and sampled them at a lower rate of $T_{s}=0.1 \mathrm{s.}{ }^{4}$

Since the coordinate frame of GNSS and V2F sensor are different, we first mapped the GNSS measurements on the Cartesian coordinate system, and then used the heading measurements from the accurate GNSS sensor as ground truth to rotate and translate the V2F measurements on the same coordinate system. This procedure allows to use the measurement models of Section III-C without further modification.

Due to the absence of an exact ground truth in this dynamic measurement scenario, measurements were considered as ground truth and IID zero-mean Gaussian measurement noise was artificially added to the GNSS measurements with $\sigma_{\mathrm{G}}^{2}=0.9216 \mathrm{~m}^{2}$ for vehicle 1 corresponding to a DGPS receiver and with $\sigma_{\mathrm{G}}^{2}=12.95 \mathrm{~m}^{2}$ for vehicle 2 corresponding to a SPS receiver. Additionally, noise was added on the V2F measurements with $\boldsymbol{Q}_{t}=0.42 \boldsymbol{I}_{2} \mathrm{~m}^{2}$ which is the worst case performance of the stereo vision camera for object positioning.

In the scene (c.f. Fig. 6), there are two pedestrians (features) standing at an intersection and vehicle 1 and 2 are driving along perpendicular roads. Due to the limited sensor FoV of the camera, features become visible at approximately $50 \mathrm{~m}$ distance. Since we had only one physical vehicle, we recorded first sensor data obtained by driving along one lane segment and afterwards from the perpendicular lane segment. The two vehicle driving scenario was then obtained by adjusting the time basis of one lane recording. Due to this hardware limitation, the proposed filter had to be run offline. Note that additional results using the same hardware and the proposed filter have been reported in [44].

\section{B. Discussion}

In Fig. 6, the trajectories of vehicle 1 (V1) and vehicle 2 (V2) are plotted together with the estimated feature and vehicle positions. Vehicle 1 moving from the lower right upwards the left of the figure, uses a DGPS receiver, and is therefore able to track the features quite accurately. In contrast, vehicle 2 , which uses a SPS receiver, cannot contribute much in accurate feature tracking, but its V2F measurements allow to transfer position information to update the vehicle state. From the figure, we observe that the estimated vehicle track is much closer to the true trajectory compared to a local KF using only GNSS measurements. As vehicle 2 approaches the intersection, its performance deteriorates and achieves the same performance as the local KF. The reason for this is that vehicle 1 has already passed all features at this point in time and cannot provide tracking information on the features. Since we assume mobile features, no significant position information towards vehicle 2 is provided after a few time steps.

In Fig. 7, the vehicle position estimation error averaged over 50 measurement realizations is plotted over time for this two vehicle experiment. Here, we can clearly observe how the performance of vehicle 2 approaches the performance

\footnotetext{
${ }^{4}$ Note that this synchronization step is not needed, but it simplified analysis of the collected measurements w.r.t. filters' estimates.
} 


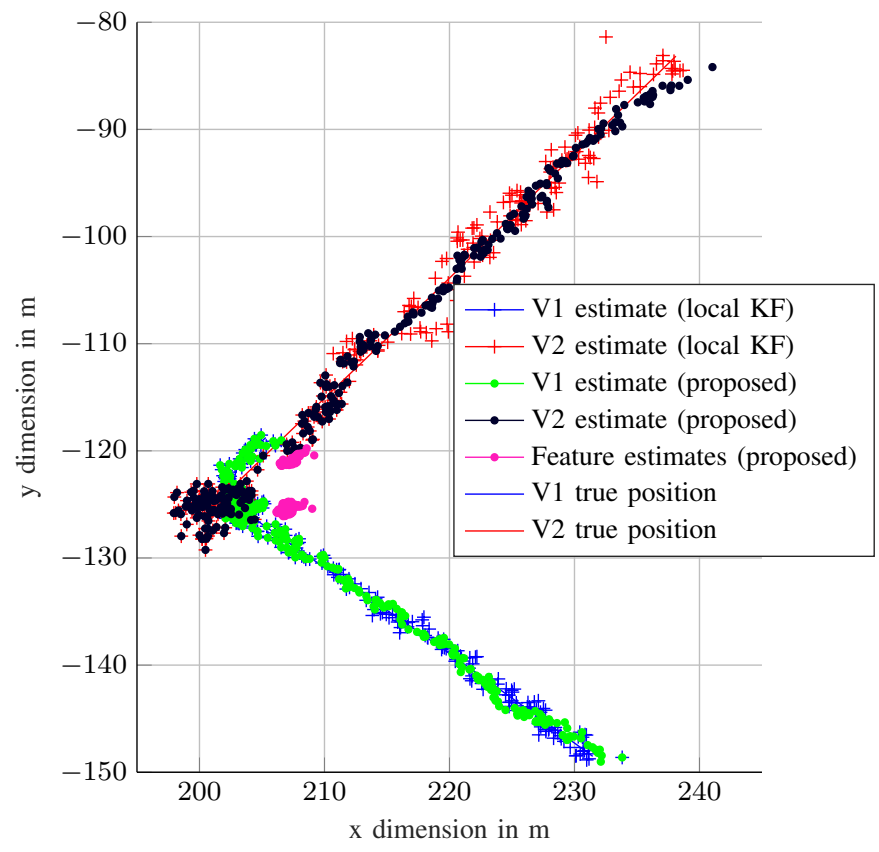

Fig. 6. Estimated vehicle (V1, V2) and feature positions using a local KF and with the proposed MTT filter for one measurement realization.

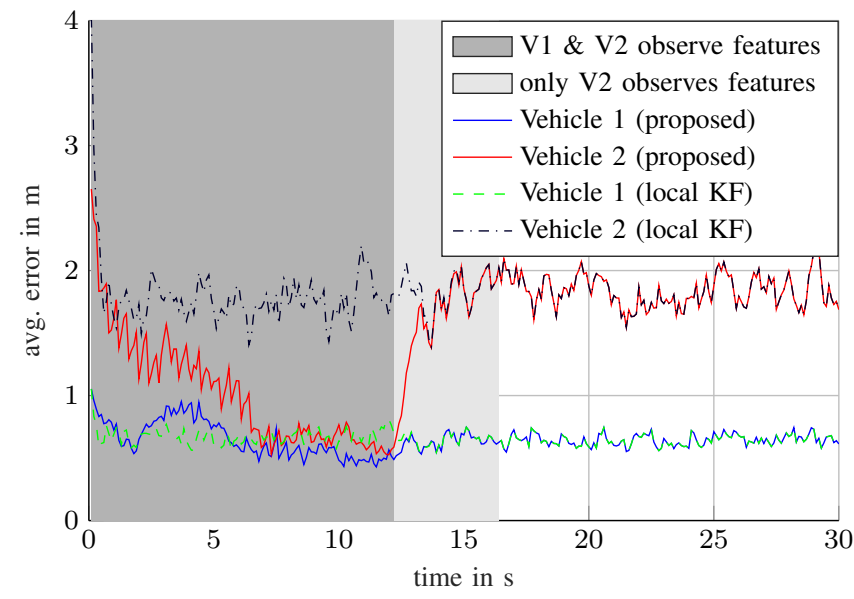

Fig. 7. Average vehicle position estimation error is over time for experimental measurement data.

of vehicle 1 when both vehicles observe the features (until approximately $12 \mathrm{~s}$ time). It then deteriorates from $12 \mathrm{~s}$ to $16 \mathrm{~s}$ due to the absence of $\mathrm{V} 2 \mathrm{~F}$ measurements from vehicle 1 . After approximately $16 \mathrm{~s}$, vehicle 2 has passed all features as well and performance of the proposed MTT filter equals the performance of the local KF. We can conclude that with the proposed multisensor MTT filter, joint feature tracking provides relevant position information towards the vehicle's position allowing to improve the positioning quality for vehicles with different/varying position accuracies.

\section{CONCLUSIONS}

This paper presented a low-complexity Poisson multiBernoulli filter to jointly track multiple features (targets) as well as the state of multiple mobile sensors. This was enabled by an approximation minimizing the Kullback-Leibler divergence. Two different kinds of sensors providing observations of the sensor state itself as well as observations of features enable accurate feature and sensor state tracking. The resulting filter incorporates the uncertain sensor state in the feature tracking task by marginalizing over the uncertain sensor state in the single feature state likelihood. Information from multiple sensors is incorporated by asynchronous update steps, executed whenever sensor measurements arrive at the central node. In doing so, data association is limited to a persensor basis. Furthermore, in a multi-vehicle scenario with varying sensor qualities, an update of the uncertain sensor state is achieved by measurements of the sensor state and by means of feature tracking. Simulation and experimental results showed that through the incorporation of measurements provided by different sensors, the feature tracking performance is superior to the track-oriented marginal multiple target multi-Bernoulli/Poisson (TOMB/P) filter which ignores sensor uncertainty, and comparable when its measurement noise is artificially increased. Furthermore, we observed in a multivehicle scenario that through joint vehicle-feature state tracking the vehicle state uncertainty can be significantly reduced compared to track the vehicle state alone. Applications of the proposed low-complexity filter involve cooperative vehicle driving scenarios when both information of the environment and the vehicle themselves are of interest. Furthermore, no direct vehicle-to-vehicle observations are needed, which makes it interesting for urban environments with applications such as extending the situational awareness beyond the visibility of the ego vehicle alone.

\section{REFERENCES}

[1] J. Leonard, J. How, S. Teller, M. Berger, S. Campbell, G. Fiore, L. Fletcher, E. Frazzoli, A. Huang, S. Karaman et al., "A perceptiondriven autonomous urban vehicle," Journal of Field Robotics, vol. 25, no. 10 , pp. 727-774, 2008.

[2] C. Cadena, L. Carlone, H. Carrillo, Y. Latif, D. Scaramuzza, J. Neira, I. Reid, and J. J. Leonard, "Past, present, and future of simultaneous localization and mapping: Toward the robust-perception age," IEEE Transactions on Robotics, vol. 32, no. 6, pp. 1309-1332, 2016.

[3] F. Meyer, O. Hlinka, H. Wymeersch, E. Riegler, and F. Hlawatsch, "Distributed localization and tracking of mobile networks including noncooperative objects," IEEE Transactions on Signal and Information Processing over Networks, vol. 2, no. 1, pp. 57-71, March 2016.

[4] G. Soatti, M. Nicoli, N. Garcia, B. Denis, R. Raulefs, and H. Wymeersch, "Enhanced Vehicle Positioning in Cooperative ITS by Joint Sensing of Passive Features," in IEEE 20th International Conference on Intelligent Transportation Systems (ITSC), Oct 2017.

[5] R. P. Mahler, Statistical multisource-multitarget information fusion. Artech House, Inc., 2007.

[6] — Advances in Statistical Multisource-Multitarget Information Fusion. Artech House, 2014.

[7] S. Mori, C.-Y. Chong, E. Tse, and R. P. Wishner, "Multitarget multisensor tracking problems. part 1. a general solution and a unified view on Bayesian approaches," Advanced Information and Decision Systems Mountain View, CA, Tech. Rep. AI/DS-TR-1048-01-REV, 1984.

[8] S. Mori, C.-Y. Chong, E. Tse, and R. Wishner, "Tracking and classifying multiple targets without a priori identification," IEEE Transactions on Automatic Control, vol. 31, no. 5, pp. 401-409, 1986.

[9] B.-N. Vo and W.-K. Ma, "The Gaussian Mixture Probability Hypothesis Density Filter," IEEE Transactions on Signal Processing, vol. 54, no. 11, pp. 4091-4104, 2006.

[10] B. N. Vo, S. Singh, and A. Doucet, "Sequential Monte Carlo methods for multitarget filtering with random finite sets," IEEE Transactions on Aerospace and Electronic Systems, vol. 41, no. 4, pp. 1224-1245, Oct 2005. 
[11] B.-T. Vo, B.-N. Vo, and A. Cantoni, "Analytic implementations of the cardinalized probability hypothesis density filter," IEEE Transactions on Signal Processing, vol. 55, no. 7, pp. 3553-3567, 2007.

[12] J. L. Williams, "Marginal multi-bernoulli filters: RFS derivation of MHT, JIPDA, and association-based MeMBer," IEEE Transactions on Aerospace and Electronic Systems, vol. 51, no. 3, pp. 1664-1687, 2015.

[13] Á. F. García-Fernández, J. L. Williams, K. Granström, and L. Svensson, "Poisson multi-Bernoulli mixture filter: direct derivation and implementation," IEEE Transactions on Aerospace and Electronic Systems, vol. 54, no. 4, pp. 1883-1901, 2018.

[14] B.-N. Vo, B.-T. Vo, and D. Phung, "Labeled random finite sets and the bayes multi-target tracking filter," IEEE Transactions on Signal Processing, vol. 62, no. 24, pp. 6554-6567, 2014.

[15] S. Reuter, B.-T. Vo, B.-N. Vo, and K. Dietmayer, "The labeled multiBernoulli filter," IEEE Transactions on Signal Processing, vol. 62, no. 12 , pp. 3246-3260, 2014.

[16] F. Meyer, T. Kropfreiter, J. L. Williams, R. Lau, F. Hlawatsch, P. Braca, and M. Z. Win, "Message passing algorithms for scalable multitarget tracking," Proceedings of the IEEE, vol. 106, no. 2, pp. 221-259, Feb 2018.

[17] F. Meyer, P. Braca, P. Willett, and F. Hlawatsch, "A scalable algorithm for tracking an unknown number of targets using multiple sensors," IEEE Transactions on Signal Processing, vol. 65, no. 13, pp. 3478-3493, 2017.

[18] H. Durrant-Whyte and T. Bailey, "Simultaneous localization and mapping: part i," IEEE robotics \& automation magazine, vol. 13, no. 2, pp. 99-110, 2006.

[19] J. Mullane, B.-N. Vo, M. D. Adams, and B.-T. Vo, "A random-finite-set approach to Bayesian SLAM," IEEE Transactions on Robotics, vol. 27, no. 2, pp. 268-282, 2011.

[20] E. Brekke, B. Kalyan, and M. Chitre, "A novel formulation of the Bayes recursion for single-cluster filtering," in Aerospace Conference, 2014 IEEE. IEEE, 2014, pp. 1-16.

[21] C. S. Lee, D. E. Clark, and J. Salvi, "SLAM with dynamic targets via single-cluster PHD filtering," IEEE Journal of Selected Topics in Signal Processing, vol. 7, no. 3, pp. 543-552, 2013.

[22] M. Üney, B. Mulgrew, and D. E. Clark, "Distributed localisation of sensors with partially overlapping field-of-views in fusion networks," in 2016 19th International Conference on Information Fusion (FUSION), July 2016, pp. 1340-1347.

[23] - "A cooperative approach to sensor localisation in distributed fusion networks.” IEEE Trans. Signal Processing, vol. 64, no. 5, pp. 1187$1199,2016$.

[24] J. Teng, H. Snoussi, C. Richard, and R. Zhou, "Distributed variational filtering for simultaneous sensor localization and target tracking in wireless sensor networks," IEEE Transactions on Vehicular Technology, vol. 61, no. 5, pp. 2305-2318, 2012.

[25] N. Kantas, S. S. Singh, and A. Doucet, "Distributed maximum likelihood for simultaneous self-localization and tracking in sensor networks," IEEE Transactions on Signal Processing, vol. 60, no. 10, pp. 50385047,2012

[26] Y. Liu, W. Wang, J. Chambers, V. Kilic, and A. Hilton, "Particle flow smc-phd filter for audio-visual multi-speaker tracking," in International Conference on Latent Variable Analysis and Signal Separation. Springer, 2017, pp. 344-353.

[27] Y. Liu, W. Wang, and Y. Zhao, "Particle flow for sequential monte carlo implementation of probability hypothesis density," in 2017 IEEE International Conference on Acoustics, Speech and Signal Processing (ICASSP). IEEE, 2017, pp. 4371-4375.

[28] Y. Liu, A. Hilton, J. Chambers, Y. Zhao, and W. Wang, "Non-zero diffusion particle flow SMC-PHD filter for audio-visual multi-speaker tracking," Proc. IEEE Int. Conf. Acoustics, Speech and Signal Processing (ICASSP), 2018

[29] Y. Liu, W. Wang, and V. Kilic, "Intensity particle flow smc-phd filter for audio speaker tracking," arXiv preprint arXiv:1812.01570, 2018.

[30] Y. Liu, V. Kılıç, J. Guan, and W. Wang, "Audio-visual particle flow smc-phd filtering for multi-speaker tracking," IEEE Transactions on Multimedia, vol. 22, no. 4, pp. 934-948, 2019.

[31] Y. Liu, Q. Hu, Y. Zou, and W. Wang, "Labelled non-zero particle flow for smc-phd filtering," in ICASSP 2019-2019 IEEE International Conference on Acoustics, Speech and Signal Processing (ICASSP). IEEE, 2019, pp. 5197-5201.

[32] Y. Liu, W. Wang, J. Chambers, V. Kilic, and A. Hilton, "Particle flow PHD filtering for audio-visual multi-speaker tracking." $\mathrm{PhD}$ diss., University of Surrey, 2019.

[33] M. Fröhle, C. Lindberg, and H. Wymeersch, "Cooperative localization of vehicles without inter-vehicle measurements," in IEEE Wireless Communications and Networking Conference (WCNC), 2018, pp. 1-6.
[34] C. M. Bishop, Pattern Recognition and Machine Learning. Springer, 2006.

[35] J. L. Williams, "Graphical model approximations of random finite set filters," arXiv preprint arXiv:1105.3298, 2011.

[36] M. S. Arulampalam, S. Maskell, N. Gordon, and T. Clapp, "A Tutorial on Particle Filters for Online Nonlinear/Non-Gaussian Bayesian Tracking," IEEE Transactions on Signal Processing, vol. 50, no. 2, pp. 174 188,2002

[37] J. A. Castellanos, J. D. Tardós, and G. Schmidt, "Building a global map of the environment of a mobile robot: The importance of correlations," in Robotics and Automation, 1997. Proceedings., 1997 IEEE International Conference on, vol. 2. IEEE, 1997, pp. 1053-1059.

[38] J. Williams and R. Lau, "Approximate Evaluation of Marginal Association Probabilities with Belief Propagation," IEEE Transactions on Aerospace and Electronic Systems, vol. 50, no. 4, pp. 2942-2959, 2014.

[39] A. A. Saucan, M. J. Coates, and M. Rabbat, "A multisensor multiBernoulli filter," IEEE Transactions on Signal Processing, vol. 65, no. 20, pp. 5495-5509, Oct 2017.

[40] J. L. Williams and R. A. Lau, "Multiple scan data association by convex variational inference," IEEE Transactions on Signal Processing, vol. 66 no. 8, pp. 2112-2127, 2016.

[41] HIGHTS project deliverable D5.2, "Specifications of implemented cooperative and fusion algorithms," Jacobs University Bremen, Tech. Rep., 2016.

[42] O. T. S. Ltd, "Datasheet RT3000 v2 GNSS/INS for high dynamic vehicle testing," Oxford Technical Solutions Ltd, United Kingdom, Tech. Rep., 2018.

[43] D. Schuhmacher, B.-T. Vo, and B.-N. Vo, "A consistent metric for performance evaluation of multi-object filters," IEEE Transactions on Signal Processing, vol. 56, no. 8, pp. 3447-3457, 2008.

[44] M. Fröhle, K. Granström, and H. Wymeersch, "Multiple target tracking with uncertain sensor state applied to autonomous vehicle data," in 2018 IEEE Statistical Signal Processing Workshop (SSP), 2018, pp. 628-632. 